\title{
An epidural blood patch for spontaneous intracranial hypotension - a case report
}

\begin{abstract}
Spontaneous Intracranial Hypotension $(\mathrm{SIH})$ is a potentially serious, rare, pathophysiological entity of diverse origin. Orthostatic headache, low CSF pressure and diffuse meningeal enhancement on brain MRI are the major features. We report a 36 year old nun with an acute onset of severe headache over the vertex of the head becoming holo-cephalic, associated with neck pain, peaking in intensity in 15-on assuming the erect posture and relieved on assuming the recumbent position. Conventional treatment resulted in minimal improvement. MRI of the brain with gadolinium showed cerebellar tonsillar descent and diffuse meningeal enhancement. Lumbar puncture revealed an opening pressure of $60 \mathrm{~mm}$ of $\mathrm{H} 2 \mathrm{O}$ and the CSF unremarkable on analysis. With the working diagnosis of $\mathrm{SIH}$, she was posted for an epidural blood patch.
\end{abstract}

After a pre-anaesthetic checkup and due consent, the patient was pre-medicated. In the operating room, the patient was positioned and draped in the left lateral position with a 15 degree trendelenburg tilt. At the L2-L3 intervertebral space, the primary anaesthesiologist identified the epidural space with an $18 \mathrm{G}$ Touhy needle adopting the loss of resistance to air technique. Simultaneously a $16 \mathrm{G}$ needle with extension was secured by the first assistant in the cephalic vein. Blood collected in $10 \mathrm{~mL}$ syringes was then introduced into the epidural space. After a volume of $26 \mathrm{~mL}$, significant lower limb parasthesia and pain was complained of. The procedure was halted and the patient placed supine. She was discharged and noted to be symptom free at follow ups.

Keywords: spontaneous intracranial hypotension, headache, epidural blood patch
Volume 4 Issue 4 - 2016

\author{
Ashish Alexander,' Shoba Philip,' Prashant \\ Varghese ${ }^{2}$ \\ 'Department of Anaesthesiology and Critical Care, India \\ ${ }^{2}$ Department of Neurology, India
}

Correspondence: Shoba Philip, Head of Department, Department of Anaesthesiology and Critical Care, Lourdes Hospital, Pachalam P.O., Kochi, Kerala, India 682012, Tel 009। 9847920355,Email dr.shobaphilip@gmail.com

Received: January 26, 2016 | Published: February 15, 2016
Abbreviations: CSF, cerebrospinal fluid; CT, computerised tomography; ECG, electrocardiogram; ICP, intracranial pressure; ICHD, international classification of headache disorders; MRI, magnetic resonance imaging; NSAID, non steroidal anti inflammatory drug; NIBP, Non invasive blood pressure monitoring; SIH, spontaneous intracranial hypotension

\section{Introduction}

Spontaneous Intracranial Hypotension (SIH) is a single, potentially serious, rare, path physiological entity of diverse origin. Orthostatic headache, low cerebrospinal fluid (CSF) pressure and diffuse meningeal enhancement on brain Magnetic Resonance Imaging (MRI) are the major features of the syndrome although some cases do have non-orthostatic chronic headache or normal CSF pressure with no evidence of meningeal enhancement. Therefore careful history taking and a high level of suspicion are the mainstay for the diagnosis. ${ }^{1}$

Amongst imaging studies, most patients with a low intracranial pressure (ICP) will have a normal brain Computerised Tomography (CT). However a brain and spine MRI with gadolinium to examine the parenchyma and meninges coupled with magnetic resonance angiography or venography are helpful in confirming the diagnosis and/or ruling out any other condition. A lumbar puncture with measurement of the CSF opening pressure and CSF analysis must be performed in all patients with new onset unusual headache when pain persists and brain imaging fails to provide a definitive diagnosis. ${ }^{1}$

The classical description of the aetiology involves CSF leakage comparable to that which occurs following an accidental dural puncture during a lumbar puncture. In both cases there is a disruption of the CSF hydrodynamics and the ability of the fluid to provide buoyancy and support to the brain and the nerve roots. With the caudal shift of the brain towards the foramen magnum complications described include: subdural hematoma, brainstem compression resulting in stupor, cranial nerve palsies and cerebellar tonsillar herniation. SIH per se can lead to cortical venous thrombosis probably because of the resultant decline in the venous blood flow velocity. ${ }^{1}$

\section{Case presentation}

We report a 36 year old right handed nun, a previously healthy woman who came to her Neurophysician after having daily headaches for a period of ten days. The acute onset of severe headache occurred while preparing for an examination. The first episode had a prelude of objective vertigo. The headache, abrupt in onset and peaking at fifteen to twenty minutes was severe in nature. The headache began over the vertex of the head and rapidly became holo-cephalic. She described no aura, visual change or neurological symptoms. Her headache was associated with a sense of heaviness as well as with neck pain and was in fact debilitating in nature. Symptoms, apparently, sometimes appeared and almost always increased in intensity on assuming the erect posture and was relieved on attaining the recumbent position. Over the last ten days the headache was also reportedly associated with nausea and was once even associated with an episode of vomiting. There was no history of recurrent headaches in the past or history suggestive of any precursors of migraine. There was neither a history of fever, skin rash, tick bites, head trauma, head surgery and the family history was negative for frequent headaches. She does have a history of low back pain since the last 3 weeks not related to any trauma though.

Workup included consultations with the institute's Otolaryngologist and Ophthalmologist. This revealed nothing remarkable. A computerized tomography (CT) of the brain done to evidence any bleed, was unremarkable. Initial treatment with a non-steroidal 
anti-inflammatory drug (NSAID) resulted in minimal improvement. Three days later, the patient went to her local hospital emergency room because of severe headache worsened by sitting or standing, as well as nausea, anorexia, and decreased fluid intake. She was then referred back to us. Physical examination revealed an ill-appearing woman with mild nuchal rigidity. Blood pressure was normal without orthostasis, and she was a febrile. General medical examination was within normal limits. Neurologic examination revealed normal cranial nerves without papilledema, and her vision was intact. Strength, sensations, and coordination were normal. There was minimal hyper reflexia, though plantar responses were flexor bilaterally. No other pathologic reflexes were elicited.

Serum chemistry values, peripheral blood total and differential counts and the erythrocyte sedimentation rate were within normal limits. Magnetic resonance imaging (MRI) of the brain with gadolinium showed cerebellar tonsillar descent suggestive of Chiari type I malformation and diffuse meningeal enhancement. Lumbar

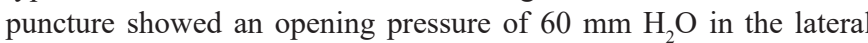
decubitus position, with cerebrospinal fluid (CSF) that was clear but drained slowly. Laboratory analysis showed a cell count of $4 / \mathrm{mm} 3$ (normal, 0 to $4 / \mathrm{mm} 3$ ), all lymphocytes, no red blood cells, protein value of $45 \mathrm{mg} / \mathrm{dL}$ (normal, 30 to $45 \mathrm{mg} / \mathrm{dL}$ ), and a glucose value of $57 \mathrm{mg} / \mathrm{dL}$ (normal, 45 to $60 \mathrm{mg} / \mathrm{dL}$ ). Results of cultures and cytologic examination were negative. A radionuclide cisternography with technetium-labeled diethylenetriamine-pentaacetic acid for the evidence of any CSF leak wasn't done because of its unavailability in our institute. The patient was admitted, placed on complete bed rest, and given high flow-rates of intravenous normal saline along with various combinations of oral and parenteral analgesics. There was limited improvement in symptoms with the orthostatic component being still significant. With the working diagnosis of SIH by the neurologist and the entire team, she was posted for an epidural blood patch as part of our contingency plan.

After a routine pre-anaesthetic checkup with emphasis on the symptomology, our patient was de-briefed in detail about the procedure and its possible outcomes. Due consent was taken, NSAIDs and Aspirin confirmed to have been stopped appropriately, coagulation profile checked to be normal and patient prepared with overnight fasting and pre-medication with anti-aspiration prophylaxis, anti-anxiety medications and maintenance intravenous fluids. On the day of the procedure, the patient was shifted to the Operating Room (OR) after an early morning repeated dosage of anti-aspiration and anti-anxiety medications.

In the Operating Room (OR), all basic monitors (Pulse Oximeter, NIBP and ECG) were attached and a pre-medication dosage of intravenous Midazolam (1mg) was administered and oxygen supplementation (at 41/min) via a Hudson's mask delivered. Our patient was then positioned, painted and draped appropriately in the left lateral position with a 15 degree trendelenburg tilt. The L2-L3 intervertebral space was manually identified using surface anatomy as a guide and local anaesthetic infiltrated at the proposed site. The primary anaesthesiologist then went on to identify the epidural space at the same level with an $18 \mathrm{G}$ Touhy needle adopting the loss of resistance to air technique. Simultaneously an 16G large bore needle with extension was secured by the first assistant in the cephalic vein under strict asepsis and free blood collected slowly with intermittent clenching of the palm by the patient to facilitate free flow. Blood hence collected in $10 \mathrm{~mL}$ syringes was introduced by the primary anaesthesiologist into the epidural space identified. As incremental volumes were added the patient was quizzed on any developing parasthesia or pain in the lumbar distribution of nerves. A total of 26
$\mathrm{mL}$ of blood was introduced when significant lower limb parasthesia and pain was complained of. The procedure was halted, sites dressed and the patient placed supine with the same trendlenburg tilt.

After an observation period of 2 hours in the recovery room the patient was shifted back to her room with the advices of remaining supine for 24hours as far as possible and to avoid all straining manoeuvres, including lifting heavy weights, for at least a month henceforth. She was symptom free for the next two day and was discharged home on the third day.

A one month follow up revealed the patient to be symptom free, happy and able to go about her assigned duties at her convent. Further long term follow ups at three and six revealed her to be symptom free and able to perform her duties at difficult areas where she had to serve as part of her religious mission. We therefore report (above) the successful management of a patient presenting with an acute onset orthostatic headache who had a clinics-radiological diagnosis of Spontaneous Intracranial Hypotension, with a single shot Lumbar epidural blood patch with relief extending into the first, third and sixth month review periods.

\section{Discussion}

Spontaneous intracranial hypotension is probably not as rare as generally thought. With an incidence of $2-5$ cases per 100000 per year, ${ }^{2,3}$ it has been reported in patients ranging from 2 years all the way to 86 years, with the peak incidence between 35 and 42 years and commoner in women..$^{2,4-6}$ At least a third of patients report a trivial trigger or trauma. ${ }^{2,7-9} \mathrm{~A}$ few patients have a history of connective tissue disorders or clinical features suggestive of it. ${ }^{10-12}$ Bariatric procedures are another possible risk factor as well as CSF leaks attributed to cervical bone disorders. ${ }^{13,14}$

\section{Clinical findings}

In patients younger than 45 years headache is the most common presenting symptom of spontaneous intracranial hypotension while in those above 45 years it may be vestibulo-cochlear manifestations like tinnitus, sound distortion and dizziness. ${ }^{1}$ The typical headache is bilateral (can be unilateral), orthostatic and very much similar to that of the post dural puncture headache. Pain intensity varies from mild to very severe even making it impossible for the patient to adopt the erect posture. Exacerbation of the pain occurs on performing a valsalva manoeuvre as well as on shaking of the head. Alternatively the headache may worsen only minutes to hours after assuming the erect posture ${ }^{15}$ or may improve without full resolution minutes or hours after assuming the supine or lateral lying positions. ${ }^{16}$ The orthostatic features of the headache may become less obvious over time. A cohort of patients with SIH may ultimately present with chronic daily headache and associated anxiety and depression. ${ }^{17}$

Rarely they may present with headache that is non-postural ${ }^{18,19}$ paradoxically caused by lying down ${ }^{20}$ or intermittently triggered by coitus, ${ }^{9}$ cough, ${ }^{21}$ head movements ${ }^{22}$ or various forms of exertion. ${ }^{23}$ The headache may be associated with nausea or emesis, neck stiffness and cochlear-vestibular signs commonly ${ }^{2,5,24-26}$ or with facial numbness, pain or weakness and dysgeusia less commonly. ${ }^{27}$ Quite rarely severe brain sagging may also cause diencephalic herniation with decreased levels of consciousness, ${ }^{28}$ encephalopathy, ${ }^{29}$ or coma. ${ }^{30}$

\section{Investigations}

A Brain CT of a SIH patient is usually normal while a Brain MRI may be normal in 20\%. Typical MRI abnormalities are often obtained with diffuse pachymeningeal gadolinium enhancement and subdural 
fluid collection (eg: Subdural hygroma). ${ }^{24,26,31-34}$ The delay between the onset of symptoms and the appearance of any MRI abnormalities are unknown. A spinal MRI might show cervical pachymeningeal enhancement or fluid non-compressive collections in the epidural space or in surrounding soft tissue near the cervical vertebra. ${ }^{35}$ A CSF study might reveal pleiocytosis (10-50 lymphocytes/micro-litre and rarely to 220 lymphocytes), a raised erythrocyte count, xanthochromia and increased protein concentrations. ${ }^{32,36}$ Invasive imaging techniques such as the intra-dural injection of contrast are reserved for cases with unidentified dural leaks not responding to two or three epidural blood patch procedures.

\section{Diagnosis}

A combination of orthostatic hypotension and pachymeningeal gadolinium enhancement in the absence of a history of a dural puncture strongly suggests a diagnosis of SIH. In view of the wide range of clinics-radiological presentations, many patients may not satisfy the ICHD diagnostic criteria. The following is a diagnostic criteria proposed by experts in 2011 (Table 1). ${ }^{37}$

Table I Diagnostic Criteria of SIH

\section{Clinico - Radiological Features}

Signs and symptoms of decreased intracranial pressure (headache,

I) nausea, vomiting, cochlear-vestibular symptoms) in an awake patient.

2) No localising focal neurologica I signs, except cranial nerve symptoms.

\section{Two of the following:}

a) Brain MRI shows signs of intra-cranial hypotension (eg: brain sagging or pachymeningeal enhancement) or is strictly normal.

3) b) Sustained improvement after epidural blood patching c) Low CSF opening pressure (less than $60 \mathrm{~cm}$ of $\mathrm{H}_{2} \mathrm{O}$ ) measured in the sitting position.

d) Demonstration of an active spinal CSF leak

4) No dural puncture within the 4 weeks preceding the headache onset.

5) Not better accounted for by another disorder

\section{Management}

Early recognition of the syndrome is essential for the appropriate management. After the initial management with bed rest, hydration, NSAIDs or Aspirin and/or Caffeine, at times, 15-30\% of cases get relief $^{5}$ but those with no relief after one week of bed rest have the treatment option of an epidural blood patch. Since many patients with compelling clinical or radiological signs of SIH have no demonstrable CSF leak, whenever headache is orthostatic and an exclusion of other causes of headache made, an epidural blood patch can be performed. ${ }^{38,39}$ The blood introduced into the epidural space increases the pressure on the dura and reverses the gradient between the CSF and the epidural veins promoting the healing of the dural rent. The overall efficiency of a blood patch in SIH is about $70 \%$ for complete immediate response and no relapse within 6 months [40-44]. When the first epidural patch is inefficient, two further patches can be done at least five days apart. Different approaches have been described, either a single site epidural patch wherein a volume of $10-80 \mathrm{ml}$ of autologous blood until severe lumbar pain or headache indicates tamponade of the epidural space or a double site (thoraco-lumbar and lower lumbar) aiming at similar end points. ${ }^{1}$ Bed rest is advised for a few hours to 24 hours in a horizontal/trendelenburg position. ${ }^{45}$ Complications include a transient lumbar pain, mild fever, mild rebound headaches frequently and arachnoididtis and aseptic meningitis rarely. ${ }^{1}$ Following discharge patients are advised to refrain from exercise or strenuous activity, valsalva manoeuvres (treat cough and constipation) and long journeys because a sudden increase in ICP or vibrations might dislodge a stable clot. Although epidural blood patches are successful, long term recurrences of spontaneous intracranial hypotension is not uncommon and is reported to be up to $25 \%$. $^{38,46}$

\section{Acknowledgements}

Dr. Shoba Philip, Department of Anaesthesiology and Critical Care.

Dr. Prashanth Varghese, Consultant Neurophysician, Department of Neurology.

\section{Conflicts of Interset}

None.

\section{References}

1. Ducros A, Biousse V. Headache arising from idiopathic changes of CSF pressure. Lancet Neurology. 2015;14(6):655-6681.

2. Schievink WI. Spontaneous spinal cerebrospinal fluid leaks and intracranial hypotension. JAMA.2006; 295(19):2286-2296.

3. Schievink WI, Morreale VM, Atkinson JL, et al. Surgical treatment of spontaneous spinal cerebrospinal fluid leaks. $J$ Neurosurg. 1998;88(2):243-246.

4. Schievink WI, Maya MM, Louy C, et al. Spontaneous intracranial hypotension in childhood and adolescence. J Pediatr. 2013;163(2):504-510

5. Chung SJ, Kim JS, Lee MC. Syndrome of cerebral spinal fluid hypovolemia: clinical and imaging features and outcome. Neurology. 2000;55(9):1321-1327.

6. Mea E, Chiapparini L, Savoiardo M, et al. Clinical features and outcomes in spontaneous intracranial hypotension: a survey of 90 consecutive patients. Neurol Sci. 2009;30(Suppl 1):S11-S13.

7. Schievink WI, Louy C. Precipitating factors of spontaneous spinal CSF leaks and intracranial hypotension. Neurology. 2007;69(7):700-702.

8. Kusnezov NA, Velani SA, Lu DC. Cerebrospinal fluid leak secondary to chiropractic manipulation. Surg Neurol Int. 2014;4(Suppl 2):S118 $\mathrm{S} 120$.

9. Giraud P, Chauvet S. Secondary pneumocephalus after lumbar puncture: an unusual complication of spontaneous intracranial hypotension? Clin Neurol Neurosurg. 2013;115(10):2204-2206

10. Mokri B, Maher CO, Sencakova D. Spontaneous CSF leaks: underlying disorder of connective tissue. Neurology. 2008;58(5):814-816.

11. Schievink WI, Gordon OK, Tourje J. Connective tissue disorders with spontaneous spinal cerebrospinal fluid leaks and intracranial hypotension: a prospective study. Neurosurgery.2004; 54(1):65-70.

12. Liu FC, Fuh JL, Wang YF, et al. Connective tissue disorders in patients with spontaneous intracranial hypotension. Cephalalgia. 2011;31(6):691-695.

13. Schievink WI, Goseland A, Cunneen S. Bariatric surgery as a possible risk factor for spontaneous intracranial hypotension. Neurology. 2014;83(20):1819-1822.

14. Eross EJ, Dodick DW, Nelson KD, et al. Orthostatic headache syndrome with CSF leak secondary to bony pathology of the cervical spine. Cephalalgia. 2002;22(6):439-443. 
15. Leep Hunderfund AN, Mokri B. Second-half-of-the-day headache as a manifestation of spontaneous CSF leak. J Neurol. 2012;259(2):306-310.

16. Schievink WI. Headache relief from lateralizing head and neck positions in spontaneous intracranial hypotension. Neurology. 2011;77(8):799-800.

17. Lobo R, Kiely P, Watts M, et al. Spontaneous intracranial hypotension presenting as a chronic daily headache. BMJ Case Rep. 2011.

18. Mea E, Franzini A, D'Amico D, et al. Treatment of alterations in CSF dynamics. Neurol Sci. 2011;32(Suppl 1):S117-S120.

19. Schievink WI, Smith KA. Nonpositional headache caused by spontaneous intracranial hypotension. Neurology. 1998;51(6):1768-1769.

20. Ferrante E, Savino A. Nonpostural headache by spontaneous intracranial hypotension. Headache. 2001;43(2):127-129.

21. Mokri B, Aksamit AJ, Atkinson JL. Paradoxical postural headaches in cerebrospinal fluid leaks. Cephalalgia. 2004;24(10):883-887.

22. Ferrante T, Latte L, Abrignani G, et al. Cough headache secondary to spontaneous intracranial hypotension complicated by cerebral venous thrombosis. Neurol Sci. 2014;33(2):429-433

23. Seok HY, Eun MY, Choi JY, et al. A typical spontaneous intracranial hypotension with a head-shaking headache. Headache. 2010;50(7):1211-1214.

24. Mokri B. Spontaneous CSF leaks mimicking benign exertional headaches. Cephalalgia. 2002;22(10):780-783.

25. Mokri B. Spontaneous CSF leaks: low CSF volume syndromes. Neurol Clin. 2004;32(10):397-422.

26. Chung SJ, Ahn JH, Lee JH, et al. Orthostatic hypacusis in a patient with CSF hypovolaemia. Cephalalgia. 2006;26(5):613-616.

27. Schievink WI, Deline CR. Headache secondary to intracranial hypotension. Curr Pain Headache Rep. 2014;18(11):457.

28. Schievink WI. Spontaneous spinal cerebrospinal fluid leaks. Cephalalgia. 2008;28(12):1345-1356.

29. Ghavanini AA, Scott CA, Chan DK, et al. Management of patients with spontaneous intracranial hypotension causing altered level of consciousness: report of two cases and review of literature. Cephalalgia. 2013;33(1):43-51.

30. Beck CE, Rizk NW, Kiger LT, et al. Intracranial hypotension presenting with severe encephalopathy. Case report. J Neurosurg. 1998;89(3):470-473.

31. Loya JJ, Mindea SA, Yu H, et al. Intracranial hypotension producing reversible coma: a systematic review, including three new cases. $J$ Neurosurg. 2012;117(3):615-628.

32. Mokri B, Piepgras DG, Miller GM. Syndrome of orthostatic headaches and diffuse pachymeningeal gadolinium enhancement. Mayo Clin Proc. 1997;72(5):400-413.
33. Mokri B, Parisi JE, Scheithauer BW, et al. Meningeal biopsy in intracranial hypotension: meningeal enhancement on MRI. Neurology. 1995;45(10):1801-1807.

34. Savoiardo M, Minati L, Farina L, et al. Spontaneous intracranial hypotension with deep brain swelling. Brain. 1990;130(Pt 7):1884-1893.

35. Beck J, Gralla J, Fung C, et al. Spinal cerebrospinal fluid leak as the cause of chronic subdural hematomas in nongeriatric patients. $J$ Neurosurg. 2014;121(6):1380-1387.

36. Yousry I, Förderreuther S, Moriggl B, et al. Cervical MR imaging in postural headache: MR signs and pathophysiological implications. AJNR Am J Neuroradiol. 2001;22(7):1239-1250.

37. Balkan II, Albayram S, Ozaras R, et al. Spontaneous intracranial hypotension syndrome may mimic aseptic meningitis. Scand J Infect Dis. 2012;44(7):481-488.

38. Schievink WI, Dodick DW, Mokri B, et al. Diagnostic criteria for headache due to spontaneous intracranial hypotension: a perspective. Headache. 2011;1(9):1442-1444.

39. Franzini A, Messina G, Chiapparini L, et al. Treatment of spontaneous intracranial hypotension: evolution of the therapeutic and diagnostic modalities. Neurol Sci. 2013;34 Suppl 1:S151-S155.

40. Starling A, Hernandez F, Hoxworth JM, et al. Sensitivity of MRI of the spine compared with CT myelography in orthostatic headache with CSF leak. Neurology. 2013;81(20):1789-1792.

41. Franzini A, Messina G, Nazzi V, et al. Spontaneous intracranial hypotension syndrome: a novel speculative physiopathological hypothesis and a novel patch method in a series of 28 consecutive patients. J Neurosurg. 2010;112(2):300-306.

42. Berroir S, Loisel B, Ducros A, et al. Early epidural blood patch in spontaneous intracranial hypotension. Neurology. 2004;63(10):1950-1951.

43. Amoozegar F, Guglielmin D, Hu W, et al. Spontaneous intracranial hypotension: recommendations for management. Can J Neurol Sci. 2003;40(2):144-157.

44. Sencakova D, Mokri B, McClelland RL. The efficacy of epidural blood patch in spontaneous CSF leaks. Neurology. 2001;57(10):1921-1923.

45. Ferrante E, Arpino I, Citterio A, et al. Epidural blood patch in Trendelenburg position pre-medicated with acetazolamide to treat spontaneous intracranial hypotension. EurJNeurol.2010;17(5):715-719.

46. Ferrante E, Arpino I, Citterio A. Is it a rational choice to treat with lumbar epidural blood patch headache caused by spontaneous cervical CSF leak? Cephalalgia. 2002;26(10):1245-1246.

47. Mokri B. Spontaneous low pressure, low CSF volume headaches: spontaneous CSF leaks. Headache. 2013;53(7):1034-1053. 